\title{
ORIGINAL ARTICLE \\ Generating Rasch-based activity of daily living measures from the Spinal Cord Injury Longitudinal Aging Study
}

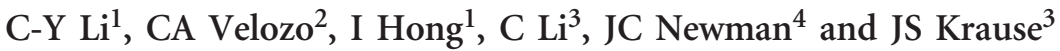

Study design: Retrospective Longitudinal Study.

Objectives: (1) To determine whether the Spinal Cord Injury Activities of Daily Living (SCI_ADL) measure shows adequate item-level and precision psychometrics; (2) to investigate whether the SCI_ADL measure effectively detects ADL changes across time; (3) to describe self-care task(s) participants can and cannot do across time.

Setting: Two Midwestern hospitals and 1 Southeastern specialty hospital in 1993.

Methods: All participants were adults with traumatic SCl of at least 1-year duration at enrollment. We used 20-year (1993-2013) retrospective longitudinal data and categorized participants into three injury levels: C1-C4 (cervical; $n=50), \mathrm{C} 5-\mathrm{C} 8$ ( $n=126)$ and T1-S5 (thoracic, lumbar and sacral; $n=168$ ). We first examined psychometrics of the SCI_ADL with factor and Rasch analyses; then we investigated longitudinal change of SCI_ADL scores at three time points over 20 years $(1993,2003$ and 2013) using generalized linear mixed modeling and post hoc analyses.

Results: The SCI_ADL measure demonstrated unidimensionality, person strata of 2.9, high Cronbach's $\alpha$ (0.93) and fair person reliability (0.76). T1-S5 had the highest measures, following C5-C8 and C1-C4 at three time points $(P<0.05)$. The C1-C4 and T1-S5 groups showed significant decreases from 2003 to 2013; however, none of the three groups showed significant differences from 1993 to $2003(P<0.05)$.

Conclusions: The SCI_ADL measure could detect longitudinal ADL changes of the population with SCl across time. The C1-C4 group decreased the most in ADLs, indicating higher need of long-term services and rehabilitation.

Spinal Cord (2018) 56, 14-21; doi:10.1038/sc.2017.99; published online 12 September 2017

\section{INTRODUCTION}

Identifying the stability of general activities of daily living (ADL), including basic $\mathrm{ADL}$ (BADL) and instrumental ADL (IADL), is central to determining aging-related functional changes after spinal cord injury (SCI). Unfortunately, there has been relatively little documentation of the long-term trends in ADL among those with SCI. Understanding the ability of individuals with SCI to perform daily activities and the pattern of recovery as they age is an essential first step to predict, monitor and improve long-term care for this population. Furthermore, understanding ADL changes over time can be predictive of long-term status of community participation for persons with SCI.

Several existing instruments are commonly used for measuring ADL function, such as the Functional Independence Measure (FIM). ${ }^{1-3}$ Earlier studies used the FIM to measure ADL function in persons with SCI. ${ }^{4-6}$ However, the FIM development was not targeted to the population with SCI. The Spinal Cord Independence Measure (SCIM), on the other hand, was designed specifically for use with persons with SCI and was later revised to address bathing, dressing, bowel management and mobility in bed through Rasch modeling resulting in SCIM II and SCIM III. ${ }^{7,8}$ The SCIM III assesses three functional areas: self-care, respiration/sphincter management and mobility. ${ }^{7,8}$
Clinicians use four basic self-care items (feeding, bathing, dressing and grooming) of the SCIM III to assess BADL function after SCI. While the SCIM is commonly used and has high clinical relevance in rehabilitation for different types of SCI levels (traumatic/nontraumatic; complete/incomplete), these four basic self-care items unlikely capture the full spectrum of essential daily functions for the individual with SCI; for example, catheter care and IADL tasks. ${ }^{9}$

Similarly, Jette et al. ${ }^{10}$ developed the Spinal Cord Injury Functional Index (SCI-FI) to capture the content of physical functioning for individuals with SCI. These authors identified five SCI-FI areas: basic mobility, fine motor function, self-care, ambulation and wheelchair mobility. Each scale consists of 36-90 items. Using a sample of 855 participants, all scales were found to be unidimensional for $80-95 \%$ of the sample. When efficiently measured with 10 items of a computerized adaptive test, the SCI-FI has shown reliability of 0.7. Moving forward, the SCI-FI seems a promising ADL instrument for the population with SCI.

When the SCI Longitudinal Aging Study was launched in 1973, similar well-established ADL instruments for individuals with SCI were not available. The SCI Longitudinal Aging Study is a 40-year project of long-term survivors from SCI that has identified changing

${ }^{1}$ Division of Rehabilitation Sciences, School of Health Professions, University of Texas Medical Branch, Galveston, TX, USA; ${ }^{2}$ Division of Occupational Therapy, College of Health Professions, Medical University of South Carolina, Charleston, SC, USA; ${ }^{3}$ Department of Health Sciences and Research, College of Health Professions, Medical University of South Carolina, Charleston, SC, USA and ${ }^{4}$ Department of Medicine, Medical University of South Carolina, Charleston, SC, USA

Correspondence: Dr JS Krause, Department of Health Sciences and Research, College of Health Professions, Medical University of South Carolina, 151-B Rutledge Avenue, B102 MSC 962, Charleston, SC 29425, USA.

E-mail: krause@musc.edu

Received 26 January 2017; revised 27 June 2017; accepted 11 July 2017; published online 12 September 2017 
patterns of health, employment and life satisfaction outcomes over time. ${ }^{11}$ Our earlier studies showed that these changing patterns include markedly declining health in terms of need for services among those who have been injured for the longest time and corresponding declines in satisfaction with health, social life, sex life and with selfrated adjustment. ${ }^{11}$ What has been noticeably absent is the measurement of change in BADL/IADL over time in this longitudinal study. As mentioned earlier, this limitation is directly related to the lack of available functional instruments relevant to the population with SCI at the early stage of the project. Thus, the newer developments of stateof-the-art measures after implementation of our longitudinal study were not applicable to our study. The absence of a self-care measure has limited the interpretability of changes in other variables and has left a void in understanding basic functional changes in the SCI Longitudinal Aging Study. Fortunately, our project included an extensive self-report assessment with a set of BADL/IADL items. ${ }^{12}$ These items serve as a promising platform to develop a functional selfcare measure that can be used to monitor participants longitudinally. A recent study also found that combining BADL and IADL items can capture self-care function with less bias than a measure with only BADL items. ${ }^{13}$

Since most SCI studies rely mainly on cross-sectional data of a relatively short time frame ( $<10$ years) across which functional changes have been measured, the SCI Longitudinal Aging Study (40 years) could provide a valuable platform to advance understanding of the aging process and further provide meaningful directions of preventive care for individuals with SCI. Although the importance of self-care function for the population with SCI has been recognized, studies about the trajectory of aging and self-care function in SCI across a substantial period of time remain relatively rare. ${ }^{6,14,15}$ Therefore, using existing BADL/IADL items to construct a functional measure could add significant value in current and future SCI studies. Successfully developing a self-care measure will lay the foundation for linking with other psychosocial, vocational and medical outcomes within this study, including all future extensions of the SCI Longitudinal Aging Study, for which a 45 -year follow-up is underway. By using Rasch modeling, we could identify whether the scale composed of existing items is unidimensional (i.e. measuring the same latent trait) and further providing person ability/item difficulty hierarchy. ${ }^{16}$ Hence, we could investigate individuals' ability to change over time.

Our purpose was to generate a Spinal Cord Injury ADL (SCI_ADL) measure using 20 years of existing data from the SCI Longitudinal Aging Study. We aimed to (1) determine whether the SCI_ADL items show adequate item-level and precision psychometrics to qualify as an ADL measure for the population with SCI, (2) to determine whether the SCI_ADL effectively detects ADL changes for three SCI levels across 20 years and (3) to describe the ADL abilities/ADL tasks participants can and cannot do across 20 years.

\section{MATERIALS AND METHODS}

\section{Participants}

Data were extracted from a larger SCI Longitudinal Aging Study, which has spanned four decades (1973-2013) and included five participant cohorts (more complete description of the methodology appears elsewhere)..$^{12}$ Each participant had sustained traumatic SCI of at least 1-year duration and was older than 18 years at the time of enrollment. This study used existing data at three time points over 20 years (those time points with most cases). Study data were collected at 10-year intervals: 1993, 2003 and 2013 from two Midwestern hospitals and one Southeastern specialty hospital in 1993 . We retrospectively reviewed 1032 participants and only included those who completed self-report assessments at all three time points and survived for 20 years from 1993 to 2013 .

\section{Instrument}

Items were extracted from the Life Situation Questionnaire-revised version ${ }^{12}$ to construct the SCI_ADL measure. The Life Situation Questionnaire-revised version was initially developed to measure a wide range of outcomes, including health and function, employment and quality of life. In developing the SCI_ADL measure, we used eight BADL items and three IADL items. It was also used to measure demographic characteristics and obtain self-reported injury levels. The BADL items were eating, dressing (upper and lower), personal hygiene, transferring, wheelchair use, catheter care, bowel management/bowel program and walking. The IADL items were driving, housekeeping and cooking. Each item had four responding options: does not apply to you (DA); independent, you need no help from another person (I); partially dependent, you need some help (PD); and dependent, someone else must do it for you (D). We converted responses into a three-point rating scale $(1=$ dependent; 2 =partially dependent; $3=$ independent $)$ for the SCI_ADL measure; thus, higher scores represent better ADL function.

The general health condition was measured based on one item of the Centers for Disease Control and Prevention self-report scale: 'How satisfied are you with the general health condition of your present life?' The rating scale ranges from 1 to 5 , with $1=$ very dissatisfied; $5=$ very satisfied. ${ }^{17}$

\section{Statistical analysis}

SAS Version 9.4., SAS Institute (Cary, NC, USA) was used to merge data across 1993, 2003 and 2013 and conduct descriptive/inferential analyses. Mplus version 7.1 was used for factor analysis including residual correlation matrix. ${ }^{18}$ Winsteps version 3.92 was used for Rasch analysis, including fits statistics, rating scale diagnoses, person strata and principal components analysis (PCA) of Rasch residuals. ${ }^{19}$ Winsteps Rasch-Welch (logistic regression) $t$-test was used to examine items with differential item functioning (DIF) across injury levels (C1-C8 vs T1-S5). The items were identified as a slight-tomoderate DIF item if the DIF contrast $\geqslant 0.43$ logits were at a significance level of $P<0.05$ and as a moderate-to-large DIF item if the DIF contrast $\geqslant 0.64$ logits were at a significance level of $P<0.05 .{ }^{20}$ If items showed DIF, the impact was tested by plotting person estimates generated from with and without DIF items using $95 \%$ confidence interval (CI). We then calculated the percent of person estimates generated from the two subsets of items beyond 95\% CI. We examined local dependency of person measure over time based on Mallinson's procedure for repeated measures..$^{21}$ Finally, we used SAS 9.4 to develop general linear models and a linear mixed model to identify main effects and post hoc analyses for between- and within-group differences across 1993-2013 with Bonferroni adjustment. ${ }^{22}$

Factor structure and item-level psychometric of SCI_ADL measure Factor structure of the SCI_ADL measure was examined using confirmatory factor analysis with the prior assumption that the SCI_ADL is a one-factor measure. We used the weighted least squares with adjustments for the mean and variance estimation. ${ }^{23}$ Four model fit statistics were used to examine the factor structure of the measure: normed $\chi^{2}$ (by dividing $\chi^{2}$ with degree of freedom to control for the effect of sample size; $\leqslant 5$ for good fit), ${ }^{24}$ TuckerLewis index ( $>0.95$ for good fit), the comparative fit index $(>0.95$ for good fit) and the root mean square error of approximation $\left(<0.06\right.$ for good fit). ${ }^{25}$ Confirmatory factor analysis was performed to examine the underlying factor structure of the scale. ${ }^{25}$

The items were identified as having local dependence problems with residual correlation coefficient $>0.2 .^{25}$ Principal components analysis was used preliminarily to examine the underlying structure pattern of the measure with the criterion of (1) the Rasch dimension (unidimensionality) explains more than $40 \%$ variance of the data, (2) residual variance from the first contrast (noise) $<5 \%$ and (3) the eigenvalue of the first contrast, which is $\leqslant 2.0 .^{19,26,27}$ In addition, we followed Smith's ${ }^{28}$ approach to compare person estimates derived from PCA first-contrast subsets of items and all the remaining items to examine whether the person estimates generated from these two sets of items differ significantly at the 0.05 level. 
Table 1 Demographic characteristics of individuals with SCI in $1993(N=344)$

\begin{tabular}{|c|c|c|c|c|c|c|c|c|c|}
\hline \multirow[t]{2}{*}{ Variables } & \multicolumn{2}{|c|}{$A / /(\mathrm{N}=344)$} & \multicolumn{2}{|c|}{$C 1-C 4(\mathrm{~N}=50 ; 15 \%)$} & \multicolumn{2}{|c|}{$C 5-C 8(N=126 ; 37 \%)$} & \multicolumn{2}{|c|}{ T1-S5 (N = 168; 49\%) } & \multirow[t]{2}{*}{$\mathrm{P}$-value } \\
\hline & $\mathrm{n}$ & $\%$ & $n$ & $\%$ & $\mathrm{n}$ & $\%$ & $\mathrm{n}$ & $\%$ & \\
\hline Gender & & & & & & & & & 0.13 \\
\hline Male & 231 & 67 & 36 & 16 & 91 & 39 & 104 & 45 & \\
\hline Female & 113 & 33 & 14 & 12 & 35 & 31 & 64 & 57 & \\
\hline Race & & & & & & & & & 0.58 \\
\hline American Indian/Alaskan Native & 5 & 2 & 0 & 0 & 2 & 40 & 3 & 60 & \\
\hline Asian & 2 & 1 & 0 & 0 & 1 & 50 & 1 & 50 & \\
\hline Black or African American & 56 & 16 & 5 & 9 & 18 & 32 & 33 & 59 & \\
\hline White & 281 & 82 & 45 & 16 & 105 & 37 & 131 & 47 & \\
\hline Hispanic origin & & & & & & & & & 0.61 \\
\hline Yes & 1 & 0 & 0 & 0 & 0 & 0 & 1 & 100 & \\
\hline No & 315 & 92 & 47 & 15 & 110 & 35 & 158 & 50 & \\
\hline Missing & 28 & 8 & 3 & 11 & 16 & 57 & 9 & 32 & \\
\hline Marital status & & & & & & & & & 0.15 \\
\hline Single & 141 & 41 & 21 & 15 & 58 & 41 & 62 & 44 & \\
\hline Married & 131 & 38 & 21 & 16 & 37 & 28 & 73 & 56 & \\
\hline Divorced & 49 & 14 & 4 & 8 & 18 & 37 & 27 & 55 & \\
\hline \multirow[t]{2}{*}{ Others } & 23 & 7 & 4 & 17 & 13 & 57 & 6 & 26 & \\
\hline & Mean & s.d. & Mean & s.d. & Mean & s.d. & Mean & s.d. & \\
\hline Age (range: $17-66$ years old) & 37.0 & 10.6 & 34.9 & 12.4 & 36.1 & 9.9 & 38.2 & 10.5 & 0.06 \\
\hline Education (year) & 14.5 & 2.9 & 13.8 & 3.1 & 14.4 & 2.9 & 14.7 & 2.8 & 0.17 \\
\hline General health score & 3.3 & 1.0 & 3.2 & 0.9 & 3.3 & 1.0 & 3.3 & 1.0 & 0.81 \\
\hline SCI_ADL raw score ${ }^{a}$ & 17.2 & 6.8 & 22.8 & 8.7 & 20.2 & 6.4 & 13.2 & 3.1 & $<0.001^{b}$ \\
\hline
\end{tabular}

Abbreviations: Min, minimum of age; max, maximum of age; SCI, spinal cord injury; SCl_ADL, Spinal Cord Injury Activities of Daily Living.

$\mathrm{SCl}$ injury level: cervical, C1-C4; cervical, C5-C8; thoracic, lumbar, sacral, T1-S5.

asignificant at $P$-value of 0.05

bLower raw SCI_ADL score means more independent.

Following the validation of unidimensionality based on confirmatory factor analysis and Rasch PCA results, we examined fit statistics of the measure using average infit mean square (MNSQ) values. Based on Smith et al. ${ }^{29}$ MNSQ calculation, the item fits the model if infit MNSQ is between 0.89 and 1.11. If items misfit, the impact of removing the misfitting items will be determined by plotting person estimates generated with and without misfitting items to examine invariance of person estimates. The percent of person estimates outside 95\% CI (based on standardized error estimated for each person estimates from both subsets of the items) will be calculated. In addition, we will calculate Spearman's correlation of person estimates generated from with and without DIF items. ${ }^{29}$ The rating scale structure was examined across three criteria: more than 10 observations of each rating category, rating step thresholds increased/decreased as the rating scale increased/decreased (monotonicity) and the outfit MNSQ for each rating category was <2.0. ${ }^{30}$ The precision of the measure was evaluated using a person strata calculation $\left(4^{\star} g+1\right) / 3$, while $g$ represents person separation index. Three strata were set as criterion for precision. ${ }^{31}$ The ceiling and floor effect was identified if more than $15 \%$ of the sample had the maximum or minimum scores. ${ }^{32}$

\section{Longitudinal ADL changes}

Participants were identified into three groups based on their injury levels: C1-C4 (cervical), C5-C8 (cervical) and T1-S5 (thoracic, lumbar and sacral). Self-care function was assessed at 10-year intervals over 20 years $(1993,2003$ and 2013). Person measures for 2003 and 2013 were anchored at mean item difficulties and rating scale step thresholds generated from the Rasch analysis of the 1993 score. We used mixed models that accounted for both random and fixed effects to analyze between- and within-group effects. We compared three mixed models with different covariance structures, including the unstructured, autoregressive and compound symmetry models. The unstructured model using unstructured covariance matrix was chosen as the final model since this model does not impose any constraints on the values. In addition, we reported effect size (ES) and Cohen's $d$ at the final year of 2013 compared with 1993 across three SCI groups to determine the estimated magnitude of difference of the SCI_ADL measure at the first and last time points. Cohen ${ }^{33}$ suggests that $0.0-0.2$ indicates a 'small' ES to indicating the standardized difference between 2 means, $0.3-0.5$ indicates 'medium' ES and $0.6-0.8$ indicates 'large' ES. Finally, since Rasch analysis places both person ability measures and item difficulty measures on the same linear continuum, a person-item map was used to compare what items participants can do across injury levels and over the 20 -year period of the study.

\section{Statement of ethics}

We certify that all applicable institutional and governmental regulations concerning the ethical use of human volunteers were followed during the course of this research.

\section{RESULTS}

\section{Participants}

Fifty individuals with $\mathrm{C} 1-\mathrm{C} 4$ injury, 126 individuals with $\mathrm{C} 5-\mathrm{C} 8$ injury and 168 individuals with T1-S5 injury survived and participated in the study over 20 years (total $N=344$ ). The mean age of the sample in 1993 was 59.5 years $($ s.d. $=11.5)$ with the majority male $(67 \%)$, White (82\%), non-Hispanic (92\%), with cervical-level injury (51\%) and single $(41 \%)$. The mean education was 14.5 years $($ s.d. $=2.9)$. The mean general health score was 3.3 (s.d. $=0.98)$ (Table 1). There were no demographic differences of the three injury groups at baseline 
(1993), including age, gender, race, Hispanic origin, marital status, education, general health score and SCI_ADL raw scores (all $P>0.05$ ) (Table 1).

\section{DIF items at two injury levels (C1-C8 vs T1-S5)}

We used a sample size of 344 for DIF testing. The Winsteps RaschWelch analysis showed seven significant DIF items between C1-C8 and T1-S5 injury levels, including dressing, catheter care, bowel management, wheelchair use, walking, housekeeping and driving (DIF contrast $\geqslant 0.64$ and $P$-value $<0.05)$. Eating had DIF contrast $\geqslant 0.64$ but was not significant; therefore, we did not consider eating as a significant DIF item (Supplementary Table 1). Four (1\%) person estimates were outside 95\% CI between with and without DIF items. Therefore, DIF items were retained in the final scale.

Factor structure and item-level psychometrics of SCI_ADL measure The confirmatory factor analysis showed that three of four fit statistics met with the criteria, indicating one dominant factor structure for the SCI_ADL measure, with normed $\chi^{2}$ (5.0) marginally meeting criterion of $5\left(\chi^{2}=222.01\right.$, d.f. $\left.=44\right)$, Comparative fit index of $0.995(>0.95)$ and Tucker-Lewis index of $0.994(>0.95)$ meeting criteria and root mean square error of approximation of $0.108(>0.06)$ not meeting criteria. All standardized factor loadings were $>0.62$ and significant at the $5 \%$ level (Supplementary Table 2). In addition, all items showed local independence with residual correlations below 0.2 (Supplementary Table 3), except one item, walking, with the residual correlations $>0.2$ with two items (transferring, -0.51 ; wheelchair use, -0.25). We decided to keep this item because it is the most difficult item in the scale. Rasch PCA met with all three criteria with the Rasch dimension explaining $61 \%$ of variance $(>40 \%)$ and first residual contrast explaining $<2 \%$ of variance with eigenvalue $<2.0$. Based on the PCA result, three items were identified as a secondary subset of the items (bowel management, catheter care and dressing) and were removed as a second set of items. However, we did not find significant differences of person estimates of these two subsets of items $(P>0.05)$. Therefore, unidimensionality is supported based on Smith's approach. ${ }^{28}$ In addition, we did not consider low fit items as a threat to the scale. Four items had high-fit values $>1.1$ (walking,

Table 2 Rasch model fit statistics of the SCI_ADL measure $(N=344$; year: 1993)

\begin{tabular}{lcccr} 
& \multicolumn{4}{c}{ Fit statistics } \\
\cline { 2 - 5 } & Measure & Model s.e. & Infit & ZSTD \\
\cline { 3 - 5 } & & & & \\
\hline Basic ADL & 3.32 & 0.11 & 1.53 & 4.8 \\
Walking & 0.65 & 0.11 & 1.66 & 5.1 \\
Transferring & 0.44 & 0.12 & 0.51 & -5.3 \\
Bowel management/bowel program & 0.17 & 0.12 & 0.62 & -3.8 \\
Catheter care & 0.01 & 0.12 & 0.44 & -6.6 \\
Dressing (upper and lower) & -0.68 & 0.12 & 0.59 & -4.4 \\
Personal hygiene & -2.06 & 0.15 & 0.55 & -3.7 \\
Eating & -2.5 & 0.17 & 1.7 & 3.8 \\
Wheelchair use & & & & \\
& & & & \\
Instrumental ADL & 0.96 & 0.11 & 0.94 & -0.5 \\
Housekeeping & 0.06 & 0.12 & 0.86 & -1.3 \\
Cooking & -0.37 & 0.12 & 1.91 & 6.7 \\
Driving & & & &
\end{tabular}

Abbreviation: SCI_ADL, Spinal Cord Injury Activities of Daily Living. transferring, wheelchair use and driving), which suggests a disturbed pattern of these four items (Table 2). To test the impact of high misfit items on person estimates, we plotted 95\% CI generated from with and without high misfit items. Only one person estimate $(1 \%)$ was beyond 95\% CI. In addition, the correlation of person estimates between the 7 -item set and the full-item set (11 items) is 0.94 . Thus, we decided to keep these four high-fit items in the final scale.

The three-point rating scale met all three rating scale criteria: each category had observation above 10, rating step thresholds and the rating scale had consistent increased/decreased patterns, and outfit MNSQ for each rating category was $<2.0$. The item difficulty levels showed that easy items (high negative measures) were wheelchair and eating, while difficult items (high positive measures) were transferring and walking, with walking 2.67 logits more difficult than transferring (Table 2). The SCI_ADL measure had person reliability of 0.76 , Cronbach's $\alpha$ of 0.93 and a precision of 2.9 person strata (which was close to the criterion of 3). Finally, the SCI_ADL measure did not have ceiling/floor effect, with 37 individuals $(11 \%,<15 \%$ of criterion) having maximum scores.

\section{Testing local dependency over time}

We compared person measures generated from the anchored and the stacked data set across three time points (1993, 2003 and 2013) and found that dependencies in the data have little effect on person measures. We provided the detailed demonstration of the anchored and the stacked measures of the first 10 persons across three time points in Figure 1.

\section{Longitudinal ADL changes}

Figure 2 presents the person measures over time for the C1-C4, C5C8 and T1-S5 groups. The group of C1-C4 had the lowest SCI_ADL scores compared with the other two groups at all three time points $(P<0.05)$. There was no significant interaction effect between group and time $(P>0.05)$, indicating no differences between the groups over time. Group was the main effect of person measure over time, indicating significant differences of person measure between groups. T1-S5 had the highest ADL function, following C5-C8 and C1-C4 at all three time points (Table 3). All two-paired comparisons across groups were significant across time $(P<0.05)$ (Table 4$)$. Within groups, time was the main effect of person measure, indicating significant differences of person measure (in this case, decreased ADL function) within individuals at all three injury levels over time. Three groups with different injury levels all showed significant selfcare function drop between 1993 and $2013(P<0.05)$. There were no significant differences between 1993 and 2003 across the three injury levels. There were significant decreases between 2003 and 2013 for the $\mathrm{C} 1-\mathrm{C} 4$ and the T1-S5 groups $(P<0.05)$, but not for the C5-C8 groups (Table 4). Between 1993 and 2013, a medium-large ES was found for $\mathrm{C} 1-\mathrm{C} 4$ (ES = 0.58; Cohen's $d=1.42$ ), a medium ES for both C5-C8 (ES =0.47; Cohen's $d=1.06)$ and T1-S5 (ES=0.46; Cohen's $d=1.03$ ) (Table 3).

Figure 2 also demonstrates the average person ability measures across different injury levels and time. Since Rasch analysis places person measures and items on the same scale, the items that an individual can do (with $>50 \%$ probability above the average person measures) and those items an individual cannot do (with $>50 \%$ probability below the average person measures) are indicated on the $y$ axis on both sides of the graph. While the C5-C8 and the T1-S5 groups are showing significant decreases in self-care ability between 1993 and 2013, the functional impact over 20 years is not marked. Over time, the T1-S5 group showed loss in ability to walk but in 2013 

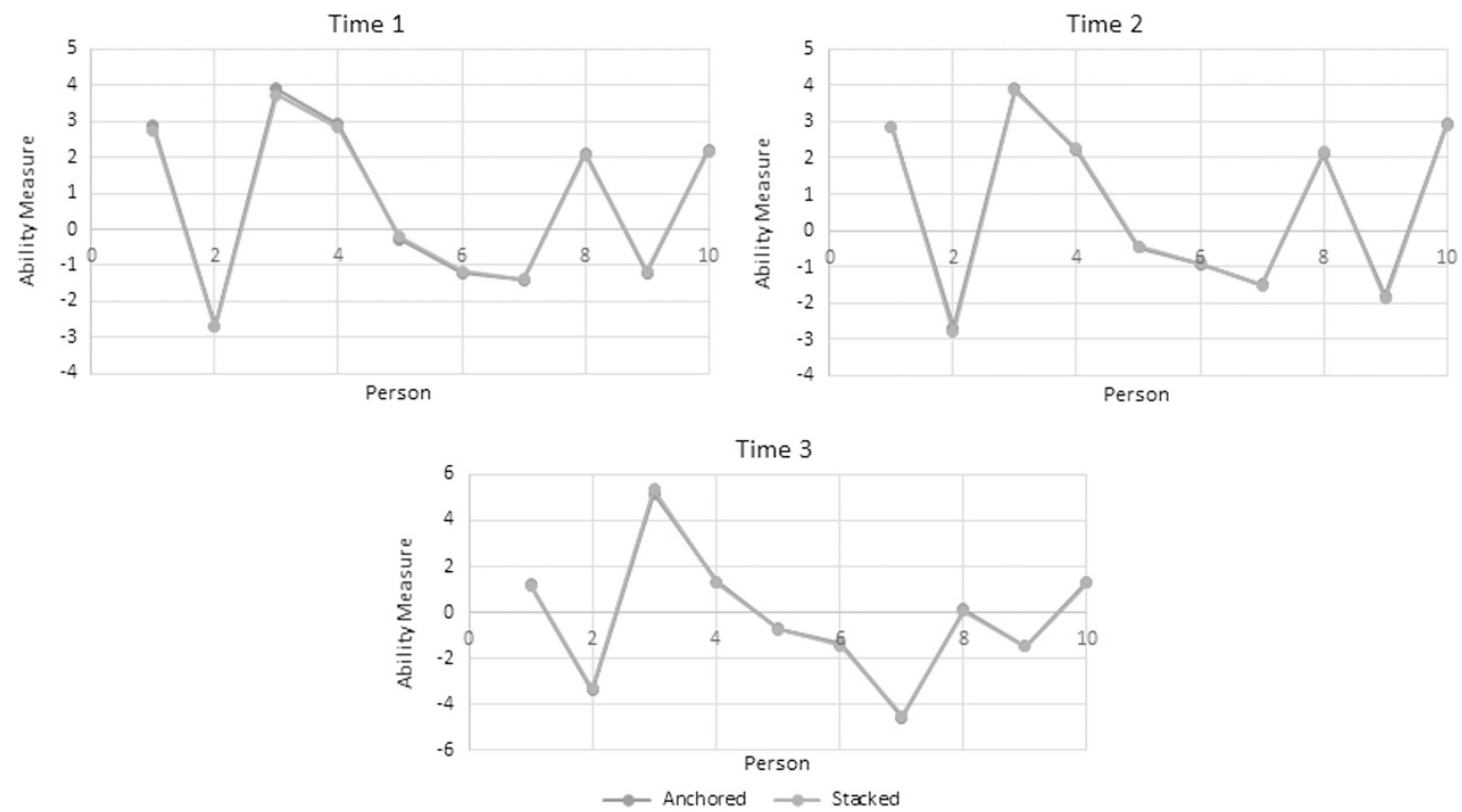

Figure 1 First 10 anchored and stacked person measures across three time points (1993, 2003 and 2013). A full colour version of this figure is available at the Spinal Cord journal online.

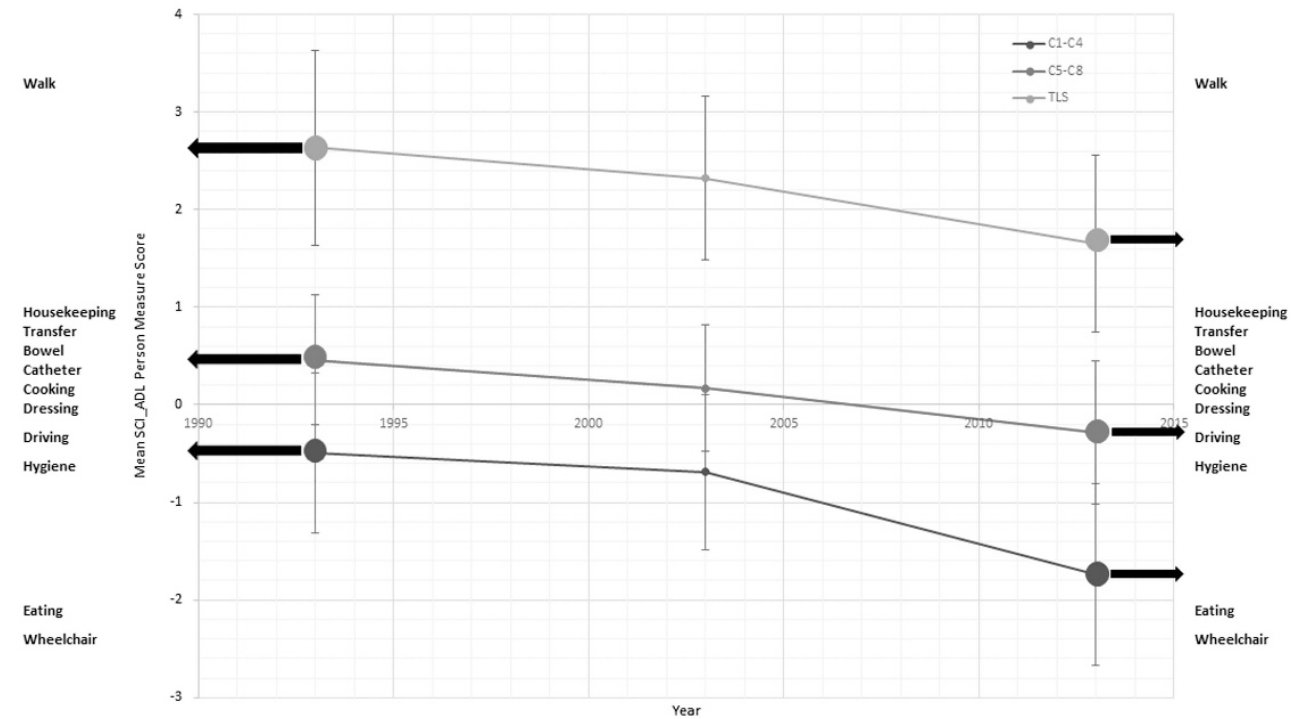

Figure 2 SCl_ADL person measure changes over time. A full colour version of this figure is available at the Spinal Cord journal online.

Table 3 SCl_ADL person measure and general health score over time (1993-2013)

\begin{tabular}{|c|c|c|c|c|c|}
\hline \multirow[t]{2}{*}{ Group } & \multirow[t]{2}{*}{$\mathrm{n}$} & \multicolumn{3}{|c|}{ Year } & \multirow[t]{2}{*}{ Effect size } \\
\hline & & 1993 & 2003 & 2013 & \\
\hline $\mathrm{C} 1-\mathrm{C} 4$ & 50 & $-0.49(0.82)^{b}$ & $-0.69(0.80)$ & $-1.74(0.93)$ & 0.58 \\
\hline C5-C8 & 126 & $0.46(0.67)$ & $0.17(0.65)$ & $-0.28(0.73)$ & 0.47 \\
\hline T1-S5 & 168 & $2.64(1.00)$ & $2.32(0.84)$ & $1.65(0.90)$ & 0.46 \\
\hline
\end{tabular}

Abbreviation: SCI_ADL, Spinal Cord Injury Activities of Daily Living.

Note: T1-S5, thoracic, lumbar and sacral.

aEffect size calculator only for 1993 and 2013.

${ }^{\mathrm{b}}$ All values presented in this table are mean person measure with standard deviation within the parentheses. were still able to perform housekeeping (Figure 2). The C5-C8 group started with the ability to maintain bladder (catheter) and bowel management and by 2003 slightly decreased in ability to cook and dress, which are lower challenge activities. In contrast, the C1-C4 group shows a dramatic drop in their self-care ability over the 20-year period. In 1993, they were able to drive and maintain hygiene but, by 2013, they were only able to eat and operate wheelchair.

\section{DISCUSSION}

The widespread use of item response theory or Rasch analysis over the past decade has led to refinements in functional outcome measures in rehabilitation. The current study successfully applied Rasch model to 
Table 4 Mean SCI_ADL person measure comparisons across group and time

\begin{tabular}{|c|c|c|c|c|}
\hline Group & Time & Group & Time & P-value \\
\hline \multirow[t]{9}{*}{$\mathrm{C} 1-\mathrm{C} 4$} & \multirow[t]{4}{*}{1993} & C5-C8 & 1993 & $0.0028^{*}$ \\
\hline & & T1-S5 & 1993 & $<0.0001^{*}$ \\
\hline & & $\mathrm{C} 1-\mathrm{C} 4$ & 2003 & 0.5997 \\
\hline & & $\mathrm{C} 1-\mathrm{C} 4$ & 2013 & $0.0011^{*}$ \\
\hline & \multirow[t]{3}{*}{2003} & C5-C8 & 2003 & $0.0070^{*}$ \\
\hline & & T1-S5 & 2003 & $<0.0001^{*}$ \\
\hline & & $\mathrm{C} 1-\mathrm{C} 4$ & 2013 & $0.0063^{*}$ \\
\hline & \multirow[t]{2}{*}{2013} & C5-C8 & 2013 & $<0.0001^{*}$ \\
\hline & & T1-S5 & 2013 & $<0.0001^{*}$ \\
\hline \multirow[t]{6}{*}{ C5-C8 } & \multirow[t]{3}{*}{1993} & T1-S5 & 1993 & $<0.0001^{*}$ \\
\hline & & C5-C8 & 2003 & 0.2223 \\
\hline & & C5-C8 & 2013 & $0.0020^{*}$ \\
\hline & \multirow[t]{2}{*}{2003} & T1-S5 & 2003 & $<0.0001^{*}$ \\
\hline & & C5-C8 & 2013 & 0.0617 \\
\hline & 2013 & T1-S5 & 2013 & $<0.0001^{*}$ \\
\hline \multirow[t]{3}{*}{ T1-S5 } & \multirow[t]{2}{*}{1993} & T1-S5 & 2003 & 0.1297 \\
\hline & & T1-S5 & 2013 & $<0.0001^{*}$ \\
\hline & 2003 & T1-S5 & 2013 & $0.0013^{*}$ \\
\hline
\end{tabular}

Abbreviation: SCl_ADL, Spinal Cord Injury Activities of Daily Living. ${ }^{*} P<0.05$.

the existing data as both a means of refining the functional measurement as the explanatory variable for change in outcomes, as well as identifying change in function over time. This current study demonstrated that developing a functional measure from an existing data set can provide a promising solution to our SCI Longitudinal Aging Study that was implemented four decades ago with a relatively non-sophisticated ADL measure.

This study showed that the SCI_ADL measure had good measurement qualities and could detect self-care functional changes across time for over a period of 20 years. Overall, self-care function decreased across time regardless of injury level. Specifically, the higher level of injury resulted in lower self-care function at three time points and across 20 years. It is worth noting that at 10 -year intervals, only groups of C1-C4 and T1-S5 had significant drops from 2003 to 2013, indicating that the decreased scores were not obvious for the first 10 years after SCI onset but were significant when the follow-up extended to 20 years. The Rasch-derived measures added to the meaning of these findings by demonstrating the impact of the decreases on ADL tasks over the 20-year period.

Few studies have generated self-care measures from existing data, even though applying a Rasch model to existing items of longitudinal studies will markedly enhance their value. McHorney ${ }^{34}$ created three modules of 31 ADL items from 4655 respondents in the Asset and Health Dynamics Among Old study using a two-parameter item response theory model. As with the present study, she was able to place all 31 items on a common scale. $\mathrm{McHorney}^{34}$ found that items involving small muscles, such as eating, were the easiest items while items involving large muscles such as walking were the most difficult items, which is consistent with the findings in the present study (e.g. eating and wheelchair use were the easiest items and walking and housekeeping were the most difficult items).

While the study by McHorney ${ }^{34}$ is the only study we could find that generated measures from the existing data, the samples were very different. The McHorney ${ }^{34}$ study involved elderly individuals, and the present study involved individuals with SCI. However, our study findings are comparable to those involving standardized measures of individuals with SCI. For example, similar to the findings in the present study, using the FIM, Bode et al. ${ }^{35}$ showed that walking and transferring were the hardest items and eating and wheelchair use were the easiest items. Similarly, Prodinger et al. ${ }^{36}$ used the Spinal Cord Independence Measure-Self Report and found moving around outdoors $100 \mathrm{~m}$ and stairs as the difficult items with eating and drinking as the easiest items.

In addition, Van Leeuwen et al. ${ }^{6}$ conducted a multicenter prospective cohort study to find predictors of life satisfaction trajectory for individuals with SCI. The participants were measured at the start of active rehabilitation, after 3 months, at discharge and at 1,2 and 5 years after discharge using the FIM. Functional independence was found as a positive predictor affecting higher life satisfaction. The SCI_ADL measure had three IADL items that may cover a wider range of daily activities compared with the FIM. Future studies may provide more evidence of advantage/limitation when incorporating IADL items in longitudinal studies for individuals with SCI. Furthermore, it would be meaningful to compare if the SCI_ADL measure, FIM, SCIM III or other SCI-targeted scales could generate comparable results in ADL function for individuals with SCI, and therefore distinguish whether there is any advantage of one instrument over another for certain scenarios.

To the authors' knowledge, this is the first study to investigate selfcare functional change for the population with SCI over a relatively long longitudinal period ( $>15$ years). The measure we developed using Rasch analysis (SCI_ADL measure) can now be used to identify differences in function over even a longer time frame, including analysis of earlier times of data collection. Owing to the advantage of the Rasch model, placing person ability and item difficulty on the same linear continuum, we can determine which functional task could or could not be accomplished over time, providing useful guidance for preventive interventions. We observed that different SCI deficits resulted in different functional decreases across similar time periods. For example, in 1993, on average, individuals with T1-S5 were close to being able to walk; individuals with $\mathrm{C} 5-\mathrm{C} 8$ injuries were able to manage catheters and bowel; while individuals with $\mathrm{C} 1-\mathrm{C} 4$ injuries were at a level of driving and maintaining hygiene. Across time, individuals with T1-TS injuries appeared to lose ability to walk, while individuals with $\mathrm{C} 1-\mathrm{C} 4$ injuries more markedly decreased in functional performance (e.g. being able to drive and maintain hygiene in 1993 but, 20 years later in 2013, only able to eat and operate wheelchairs). To target specific needs for each injury group, we suggest providing assistant or device training (for personal hygiene) for the C1-C4 group; upper body training (for transferring/housekeeping) for the C5-C8 group; and lower-extremity strength/endurance training (for walking) for the T1-S5 group.

The functional changes observed with aging underscore the importance of re-evaluation of function over time after SCI, including needs for services and safety risk. Those who have non-cervical injuries run the risk of losing their ambulatory abilities. The situation for those with high cervical injuries may be more profound, as those who have been relatively independent with aspects of their care are likely to become more dependent over time. There likely would be an elevated risk of injury as functional losses increase, unless there are additional supports, such as rehabilitation services or an increase in personal care assistants. From a policy standpoint, there will be needs for increased services to avoid functional decline. For instance, expanding insurance coverage to provide regular evaluations with aging, perhaps even every 5 years, would help individuals identify and compensate for functional 
decline. The provision of additional adaptive equipment, combined with more frequent evaluations, could be used to help individuals maintain their level of function or to compensate for changes in function. These types of policy changes may ultimately be required at a federal level, since a large portion of people with SCI are unemployed and therefore receiving Medicaid. Preserving function may ultimately lead to fewer hospitalizations and lower costs.

Our study also pointed out that different long-term care and treatment goals should be targeted dependent on injury level. SCI is a potentially explanatory factor for the greatest decline being observed among those with the highest cervical injuries. This is an important issue for future research. It is also important to go beyond simply asking for more services, or the allocation of more funds for services, but rather to identify which changes are most likely to occur next as individuals with SCI age and to target resources to prevent or delay those functional changes across time.

In summary, the SCI_ADL measure could serve as a useful tool to study self-care functional changes across time and provide practical treatment guidance for the practitioners.

\section{Limitations}

Since this study only included individuals who survived across 20 years, the result will be biased, as survivors may have higher functional levels or be healthier in general. Without question, those who are healthier have greater life expectancies, ${ }^{37}$ so the current cohort is selective in terms of relating to help the survivors. Second, the group of C1-C4 had a small sample size $(n=50)$, which decreases statistical power. In addition, the impact of technology may influence self-care function. For instance, phone- and computer-related technology changed rapidly across the past 20 years, and it is not clear whether the SCI_ADL can capture those changes (e.g. participants are likely to become more independent because of the advance of technology). Also, we did not control for length of time post injury at baseline and the neurological completeness (e.g. AIS levels) since the data are not available. However, we did not find significant group difference of demographics. Future study of this kind could compare age-graded or post-injury trajectories based on intraindividual changes in a cohort and included neurological completeness levels. In addition, we did not compare DIF items among three lesion levels because of small sample size of C1-C4 group and limited power. With regard to explaining reasons caused by different trajectories across three injury levels, the small sample size could be one factor for the identification of 10-year declines in function for two of the groups (C1-C4, non-cervical), but not the third (C5-C8). We are also aware that the level of lesion determines the ability to perform dressing. Thus, using one dressing item (combining with upper and lower body) may inaccurately reflect the respondents' dressing ability.

Finally, we did not control for neurological completeness of SCI because of sample size among those with high cervical injuries and the difficulty of measuring this construct with self-report. Without controlling for neurologic completeness, each of our groups may have been more heterogeneous in terms of function at baseline, further adding error to our measure when function declined. Because neurologic completeness of injury is related to risk of mortality, those with incomplete injuries would be more likely to be those who survived to follow-up. However, this diversity of function may also have eliminated some floor effects for function that would otherwise be observed with those with the highest injury levels. This clearly is an issue for further research.

\section{CONCLUSION}

The present study demonstrated using existing data set to generate a feasible functional measure to capture self-care trajectory change over a 20-year period for the population with SCI. While all groups showed significant drops in SCI_ADL measure over time, SCI level had significant impact on self-care function across time. Higher injury levels were associated with lower self-care function at all three time points. These initial findings have implication for long-term supports and preventive interventions to assist individuals with SCI, especially those with high cervical injuries.

\section{DATA ARCHIVING}

There were no data to deposit.

\section{CONFLICT OF INTEREST}

The authors declare no conflict of interest.

\section{ACKNOWLEDGEMENTS}

The contents of this publication were developed under a grant from the National Institute on Disability, Independent Living and Rehabilitation Research (NIDILRR; grant number 90IF0015). NIDILRR is a Center within the Administration for Community Living (ACL), Department of Health and Human Services (HHS). The contents of this publication do not necessarily represent the policy of NIDILRR, ACL, HHS, and you should not assume endorsement by the Federal Government.

1 Granger CV, Hamilton BB, Keith RA, Zielezny M, Sherwin FS. Advances in functional assessment for medical rehabilitation. Top Geriatr Rehabil 1986; 1: 59-74.

2 Grey N, Kennedy P. The Functional Independence Measure: a comparative study of clinician and self-ratings. Paraplegia 1993; 31: 457-461.

3 Heinemann AW, Linacre JM, Wright BD, Hamilton BB, Granger C. Prediction of rehabilitation outcomes with disability measures. Arch Phys Med Rehabil 1994; 75 : 133-143.

4 Fortin CD, Voth J, Jaglal SB, Craven BC. Inpatient rehabilitation outcomes in patients with malignant spinal cord compression compared to other non-traumatic spinal cord injury: a population based study. J Spinal Cord Med 2015; 38: 754-764.

5 Herzer KR, Chen Y, Heinemann AW, Gonzalez-Fernandez M. Association between timeto-rehabilitation and outcomes following traumatic spinal cord injury. Arch Phys Med Rehabil 2016; 97: 1620-1627.

6 van Leeuwen CM, Post MW, van Asbeck FW, van der Woude LH, de Groot S, Lindeman E. Social support and life satisfaction in spinal cord injury during and up to one year after inpatient rehabilitation. J Rehab Med 2010; 42: 265-271.

7 Catz A, Itzkovich M, Steinberg F, Philo O, Ring H, Ronen J et al. The Catz-Itzkovich SCIM: a revised version of the Spinal Cord Independence Measure. Disabil Rehabil 2001; 23: 263-268.

8 Itzkovich M, Gelernter I, Biering-Sorensen F, Weeks C, Laramee MT, Craven BC et al. The Spinal Cord Independence Measure (SCIM) version III: reliability and validity in a multi-center international study. Disabil Rehabil 2007; 29: 1926-1933.

9 Ullrich PM, Spungen AM, Atkinson D, Bombardier CH, Chen Y, Erosa NA et al. Activity and participation after spinal cord injury: state-of-the-art report. J Rehabil Res Dev 2012; 49: 155-174.

10 Jette AM, Tulsky DS, Ni P, Kisala PA, Slavin MD, Dijkers MP et al. Development and initial evaluation of the Spinal Cord Injury-Functional Index. Arch Phys Med Rehabil 2012; 93: 1733-1750.

11 Krause JS, Newman JC, Clark JM, Dunn M. The natural course of spinal cord injury: changes over 40 years among those with exceptional survival. Spinal Cord 2017; 55: 502-508.

12 Krause JS, Clark JM, Saunders LL. SCI Longitudinal Aging Study: 40 years of research. Top Spinal Cord Inj Rehabil 2015; 21: 189-200.

13 LaPlante MP. The classic measure of disability in activities of daily living is biased by age but an expanded IADL/ADL measure is not. J Gerontol B 2010; 65: 720-732.

14 van den Berg-Emons RJ, Bussmann JB, Haisma JA, Sluis TA, van der Woude LH, Bergen MP et al. A prospective study on physical activity levels after spinal cord injury during inpatient rehabilitation and the year after discharge. Arch Phys Med Rehabil 2008; 89: 2094-2101.

15 Hetz SP, Latimer AE, Buchholz AC, Martin Ginis KA, Group S-SR. Increased participation in activities of daily living is associated with lower cholesterol levels in people with spinal cord injury. Arch Phys Med Rehabil 2009; 90: 1755-1759.

16 Bond TG, Fox CM. Applying the Rasch Model: Fundamental Measurement in the Human Sciences, 2nd edn. Lawrence Erlbaum Associates: Mahwah, NJ, USA, 2007. 
17 Krause JS. Dimensions of subjective well-being after spinal cord injury: an empirical analysis by gender and race/ethnicity. Arch Phys Med Rehabil 1998; 79: 900-909.

18 Muthen LK, Muthen BO. Mplus User's Guide 7th edn. Muthen \& Muthen: Los Angeles, CA, USA, 2015.

19 Linacre JM. A User's Guide to Winsteps Ministeps 3.70.0: Rasch Model Computer Programs. Winsteps: Chicago, IL, USA, 2012.

20 Zwick R, Thayer DT, Lewis C. An empirical bayes approach to mantel-haenszel DIF analysis. J Ed Meas 1999; 36: 1-28.

21 Mallinson T. Rasch analysis of repeated measures. Rasch Meas Trans 2011; 25: 1317.

22 SAS Institute Inc. SAS for Windows, 9.4 edn. SAS Institute: Cary, NC, USA, 2015.

23 Revicki DA, Cook KF, Amtmann D, Harnam N, Chen WH, Keefe FJ. Exploratory and confirmatory factor analysis of the PROMIS pain quality item bank. Qual Life Res 2014; 23: 245-255.

24 Schumacker RE, Lomax RG. A Beginner's Guide to Structural Equation Modeling, 2nd edn. Lawrence Erlbaum Associates: Mahwah, NJ, USA, 2004.

25 Reeve BB, Hays RD, Bjorner JB, Cook KF, Crane PK, Teresi JA et al. Psychometric evaluation and calibration of health-related quality of life item banks: plans for the Patient-Reported Outcomes Measurement Information System (PROMIS). Med Care 2007; 45(Suppl 1): S22-S31.

26 Linacre JM. Rasch model estimation: further topics. J Appl Meas 2004; 5: 95-110.

27 Linacre JM. Predicting responses from Rasch measures. J App/ Meas 2010; 11: $1-10$.
28 Smith EV Jr. Detecting and evaluating the impact of multidimensionality using item fit statistics and principal component analysis of residuals. J Appl Meas 2002; 3: 205-231.

29 Smith RM, Schumacker RE, Bush JM. Using item mean squares to evaluate fit to the Rasch model. J Outcome Meas 1998; 2: 66-78.

30 Linacre JM. Optimizing rating scale category effectiveness. J Appl Meas 2002; 3: 85-106.

31 Wright BD, Masters GN. Number of person or item strata. Rasch Meas Trans 2002; 16 888.

32 McHorney CA, Tarlov AR. Individual-patient monitoring in clinical practice: are available health status surveys adequate? Qual Life Res 1995; 4: 293-307.

33 Cohen J. Statisical Power Analysis for the Behavioral Sciences, 2nd edn. Erlbaum: Hillsdale, NJ, USA, 1988

34 McHorney CA. Use of item response theory to link 3 modules of functional status items from the Asset and Health Dynamics Among the Oldest Old study. Arch Phys Med Rehabil 2002; 83: 383-394.

35 Bode RK, Heinemann AW, Kozlowski AJ, Pretz CR. Self-scoring templates for motor and cognitive subscales of the FIM instrument for persons with spinal cord injury. Arch Phys Med Rehabil 2014; 95: 676-9 e5.

36 Prodinger B, Ballert CS, Brinkhof MW, Tennant A, Post MW. Metric properties of the Spinal Cord Independence Measure-Self Report in a community survey. J Rehabil Med 2016; 48: 149-164.

37 Krause JS, Carter RE, Pickelsimer E, Wilson D. A prospective study of health and risk of mortality after spinal cord injury. Arch Phys Med Rehabil 2008; 89. 1482-1491.

Supplementary Information accompanies this paper on the Spinal Cord website (http://www.nature.com/sc) 